\title{
Impacto de la aplicación de medidas de humanización en unidades de hemodiálisis
}

\author{
Ana Casaux-Huertas ${ }^{1,2}$, Juana Elisabeth Cabrejos-Castilloㄹ, Nuria Pascual-Aragonés ${ }^{1}$, Marta Moreda-Díaz- \\ Pavón $^{1}$, Eva María Carrera-Rodríguez ${ }^{1}$, David Hernán-Gascueña ${ }^{1}$ \\ ${ }^{1}$ Fundación Renal Íñigo Álvarez de Toledo. Madrid. España \\ ${ }^{2}$ Escuela de Enfermería Fundación Jiménez Díaz-Campus Villalba. Madrid. España
}

Como citar este artículo:

Casaux-Huertas A, Cabrejos-Castillo JE, Pascual-Aragonés N, Moreda-Díaz-Pavón M, Carrera-Rodríguez EM, Hernán-Gascueña

D. Impacto de la aplicación de medidas de humanización en unidades de hemodiálisis.

Enferm Nefrol. 2021 Jul-Sep;24(3):279-93

\section{Resumen}

Introducción: El papel de la Enfermería Nefrológica en el cuidado y atención humanizada de los pacientes en hemodiálisis es fundamental.

Objetivos: Analizar la satisfacción percibida por pacientes y profesionales sanitarios de unidades de hemodiálisis tras la implementación de un programa para potenciar la humanización de la asistencia.

Material y Método: Estudio analítico longitudinal prospectivo cuasiexperimental con grupo control histórico. La recogida de datos se realizó en un periodo de 13 meses en 16 centros de la Fundación Renal Íñigo Álvarez de Toledo, a un total de 667 pacientes y 180 trabajadores. Se emplearon dos cuestionarios autoadministrados, uno para pacientes y otro para profesionales.

Resultados: Tras la implantación del programa, el número de pacientes que considera que el personal siempre les informa sobre los procedimientos aumentó de forma significativa $(p=0,043)$ y un $62,4 \%$ de los pacientes valoró como importante que pueda entrar algún

\section{Correspondencia:}

Ana Casaux-Huertas

E-mail: anacasaux@gmail.com familiar o persona cercana durante la sesión. Tras la implantación del proyecto, el 49,2\% de los profesionales sanitarios consideraron siempre necesaria la formación en el ámbito de la humanización y opinaban que sería conveniente mejorarla ( $p>0,005)$.

Conclusiones: Se ha advertido un incremento significativo en la satisfacción de los pacientes respecto a la información proporcionada por los profesionales y la cercanía de éstos. Se han verificado valores muy positivos respecto al trato humanizado que reciben los pacientes y su comodidad durante las sesiones de hemodiálisis. Por otra parte, los profesionales consideran necesaria la formación en el ámbito de la humanización y creen que sería conveniente mejorarla en las unidades.

PALABRAS CLAVE: humanización; asistencia sanitaria; cuidados; hemodiálisis; enfermera.

\section{Impact of the application of humanising measures in hemodialysis units}

\section{Abstract}

Introduction: The role of nephrological nursing in the humanizing care and attention of patients on hemodialysis is essential. 
Objectives: To analyse the perceived satisfaction of patients and healthcare professionals in hemodialysis units after the implementation of a humanizing program.

Material and Method: Quasi-experimental prospective longitudinal analytical study with a historical control group. Data collection was carried out during 13 months in 16 dialysis units of the Fundación Renal Íñigo Álvarez de Toledo with a total of 667 patients and 180 workers. Two self-administered Likert-type questionnaires were used, one for patients and another for professionals.

Results: After the implementation of the programme, the number of patients who consider that the staff always informs them about the procedures increased significantly $(p=0.043)$ and $62.4 \%$ of the patients valued the fact that a family member or close person can enter during the session as something valuable. After the implementation of the project, $49.2 \%$ of the healthcare professionals considered that training in the field of humanisation was always necessary and thought that it would be advisable to improve it $(p>0.005)$.

Conclusions: There has been a significant increase in patient satisfaction about the information provided by the professionals and their proximity. Very positive values have been verified regarding the humanising care received by patients and their comfort during hemodialysis sessions. On the other hand, the professionals consider training in the field of humanisation to be necessary and believe that it would be advisable to improve it in the units.

KEYWORDS: humanisation; healthcare; care; hemodialysis; nurse.

\section{Introducción}

El papel de la Enfermería Nefrológica abarca aspectos tanto técnicos como aquellos que hacen referencia al conocimiento y a la atención psicosocial, es decir, cuidar la parte humana de los pacientes considerando a la persona con todas sus necesidades, en un abordaje global ${ }^{1}$. Una competencia intrínseca de cualquier buen profesional de la enfermería es aportar cuidados holísticos y de calidad ${ }^{2,3}$. Las personas sometidas a Tratamiento Renal Sustitutivo (TRS) están en una situación de gran vulnerabilidad y necesitan el apoyo y la cercanía de los que le rodean y especialmente, de los profesionales que le cuidan y con quienes comparten mucho tiempo en el centro al que acude para recibir tratamiento.

Las unidades de hemodiálisis son lugares altamente tecnificados con máquinas complejas, que en algún sentido, podrían mecanizar los cuidados y con ello distanciar o transformar las relaciones sociales, restando humanidad a la interacción entre sujetos. Este es un riesgo que se debe evitar, la tecnología no puede suponer una barrera para que el paciente reciba un trato cercano y humano ${ }^{3}$.

En general, durante las sesiones de diálisis se producen períodos en que los pacientes permanecen hemodinámicamente estables y no precisan de muchas intervenciones desde el punto de vista asistencial, tiempo que puede dedicarse a otros cuidados más relacionados con una asistencia humanizada ${ }^{4-7}$.

La importancia de unos cuidados por parte de los profesionales que hagan énfasis en aspectos humanos radica en brindar a las personas que sufren una enfermedad, y una alteración en su vida cotidiana, la oportunidad de sentirse acompañados y apoyados para enfrentar el proceso de enfermedad6. Es decir, se debe considerar la humanización como una parte inherente al abordaje profesional de los sanitarios y como se refleja en el Plan de Humanización de la Asistencia Sanitaria 2016-2019 de la Comunidad de Madrid, "el proceso de humanización es una parte fundamental de la calidad del servicio que se presta, es un compromiso activo y una responsabilidad de cada uno de nosotros ${ }^{1 / 5}$.

La Fundación Renal Íñigo Álvarez de Toledo desarrolló en el año 2019 un proyecto pionero de Humanización en Hemodiálisis que implantó en todas sus unidades a nivel nacional. Este proyecto, está formado por una serie de líneas estratégicas encaminadas a mejorar la humanización en las unidades de hemodiálisis a todos los niveles, de forma tal que repercutan en una mejora en los cuidados integrales de los pacientes renales. De la evaluación tras la aplicación de este Proyecto de Humanización se desprenden los resultados plasmados en el presente artículo, cuyo objetivo principal es analizar la satisfacción percibida de los pacientes y los profesionales sanitarios de las unidades de la Fundación Renal Íñigo Álvarez de Toledo tras la implementación de estas medidas de humanización que se detallarán más adelante en el desarrollo del trabajo. 


\section{Material y Método}

Como hipótesis de trabajo se planteó que la mejora en los cuidados de salud, aplicando medidas de humanización en las unidades de hemodiálisis, repercutiría en una mayor satisfacción percibida de los pacientes renales. Para ello se diseñó un estudio analítico, longitudinal, prospectivo, cuasiexperimental, con grupo control histórico realizado a pacientes con Enfermedad Renal Crónica Terminal en tratamiento con hemodiálisis (HD). La recogida de datos se llevó a cabo en un periodo de 13 meses, comprendido entre los meses de agosto de 2019 y septiembre de 2020 , en las 16 Unidades de Diálisis de la Fundación Renal Iñigo Álvarez de Toledo (FRIAT) en España, incluyendo en el estudio a todos aquellos pacientes que recibían tratamiento en sus unidades de hemodiálisis y que no presentaban deterioro cognitivo u otro tipo de limitaciones para responder adecuadamente a los cuestionarios.

Se partió de una población total de aproximadamente 1.000 pacientes de los cuales tras la aplicación de dichos criterios de inclusión se obtuvo la muestra final del estudio, 667 pacientes. La selección y reclutamiento de pacientes se efectuó a través de un muestreo de oportunidad que permitió un mayor número de participantes, excluyendo únicamente a aquellos individuos que no cumplían con los criterios de selección anteriormente citados o aquellos otros que se negaron a participar en el estudio.

Previo a la implantación de las medidas de humanización, en el mes de junio de 2019 se impartió una formación en humanización a los profesionales sanitarios a través de una jornada de 5 horas. Al finalizar la misma se les explicó el Proyecto de Humanización y se pasaron los cuestionarios al personal y a los pacientes renales de las 16 unidades de hemodiálisis que participaron en el proyecto (controles históricos). Seguidamente se iniciaron las intervenciones y medidas encaminadas a mejorar la humanización de los cuidados en las hemodiálisis. Transcurridos 13 meses se volvió a pasar, tanto a pacientes como a profesionales, el mismo cuestionario del momento cero y se valoró la eficacia de la intervención (comparación del efecto de la intervención con el control histórico).

Las intervenciones que se implementaron para potenciar la humanización de las unidades de hemodiálisis se reflejan en los anexos 1 y 2.

Tras una extensa búsqueda bibliográfica en la que no se obtuvieron resultados acerca de la existencia de escalas o cuestionarios para valorar la humanización en las unidades de hemodiálisis, se diseñaron y validaron dos cuestionarios ad hoc elaborados por un panel de expertos que consensuaron los cuestionarios (con una escala de respuesta tipo likert) que se consideraron definitivos tras ser sometidos a una prueba piloto.

Ambos cuestionarios fueron autoadministrados, el cuestionario 1 "valoración de los pacientes de la atención humanizada" (anexo 3) está compuesto por 23 preguntas y el cuestionario 2 para la "valoración de los profesionales de la atención humanizada" compuesto por 11 preguntas (anexo 4).

El proyecto fue aprobado por el Comité Ético de Investigación Clínica del Hospital Universitario Fundación Alcorcón.

Los cuestionarios se cumplimentaron anónimamente previo consentimiento informado, tanto verbal como escrito por parte de los participantes, mediante una cláusula incluida en los mismos. Se distribuyeron a los pacientes durante la sesión de hemodiálisis, con la opción de cumplimentarlos en el momento o llevarlos a su domicilio para después ser entregados en un sobre cerrado al personal del centro.

Las variables de estudio fueron las siguientes:

- Variables sociodemográficas de los pacientes: edad, sexo, estado civil, tiempo en diálisis, percepción del estado de salud.

- Variables sociodemográficas de los profesionales: edad, sexo, tiempo de experiencia en diálisis, categoría profesional.

- Valoración de los pacientes de la atención humanizada: variable cualitativa o categórica ordinal obtenida a través del cuestionario 1

- Valoración de los profesionales de la atención humanizada: variable cualitativa o categórica ordinal obtenida a través del cuestionario 2 .

Para llevar a cabo el análisis estadístico de los resultados se realizó previamente la transcripción de los datos obtenidos en los cuestionarios y la codificación de las variables. De esta forma, se llevó a cabo un análisis descriptivo de las variables cuantitativas, calculando los valores medios y su desviación típica, para un intervalo de confianza del 95\%.

Para el análisis de los cuestionarios de pacientes (variables cualitativas), se asignó una puntuación a cada una de las preguntas, entre 1 y 3 , que correspondió a las contestaciones de "Nunca", "Alguna vez" y "Siempre". Con 
estos valores se calculó la media y la desviación estándar pre y post, para después realizar una comparación estadística mediante el test de la $\mathrm{t}$ de Student.

De manera similar se actuó en el cuestionario del personal, donde cada una de las respuestas recibió una calificación entre 1 y 6 que correspondió a las contestaciones de "Nunca", "Poco frecuente", "Alguna vez", "Frecuentemente", "Muy frecuentemente" y "Siempre". Se calculó la media y la desviación estándar pre y post para después realizar una comparación estadística mediante el test de la $t$ de Student. Se consideró un $p$ valor inferior a 0,05 como estadísticamente significativo.

\section{Resultados}

La muestra final estuvo compuesta por 667 pacientes, mayoritariamente hombres $(63,3 \%)$, con una edad media de $67 \pm 14,1$ años, casados o en pareja $(60 \%)$ y que llevan en tratamiento con hemodiálisis una media de 4,2 $\pm 4,3$ años.

Por lo que se refiere a la satisfacción percibida de los pacientes, cabe destacar que las categorías donde se observó una diferencia estadísticamente significativa tras la implantación de las medidas de humanización son las siguientes:

- Los pacientes que consideran que el personal siempre conoce sus gustos y preferencias aumentó de forma estadísticamente significativa de un $70,9 \%$ hasta un $79,5 \%$ de los encuestados $(p=0,015)$.

- El número de pacientes que estiman que el personal siempre les explica o informa sobre los procesos que se realizan en la diálisis aumentó de un $76,5 \%$ hasta un $81,5 \%(p=0,043)$ tras las intervenciones.

- Igualmente, los pacientes opinan en un 93,1\% frente a $87,7 \%(p=0,005)$ que siempre obtienen la información necesaria cuando le preguntan al personal sanitario de sus unidades, y que el personal se interesa y se preocupa por conocer sugerencias para mejorar el bienestar de los pacientes $(64,9 \%$ versus 52,$4 ; p=0,001)$.

Respecto a los resultados obtenidos de los profesionales, la muestra final estuvo formada por 180 trabajadores de los centros de la FRIAT, entre ellos y ordenados de mayor a menor participación: enfermeras, auxiliares de enfermería, grupo de apoyo al paciente (psicólogos, trabajadores sociales, graduados en actividad física) y médicos. La muestra estu- vo compuesta predominantemente por mujeres $(85,5 \%)$ con una edad media de $38,9 \pm 9,9$ años y con una experiencia en diálisis de 10,3 $\pm 9,2$ años.

Tras la implantación en los centros de las medidas de humanización, la pregunta "¿Hablas con los pacientes sobre acceso vascular, dieta, cuidados, etc., de forma regular?" registró un aumento en las respuestas positivas y una disminución de las negativas de forma significativa $(p=0,005)$ (gráfico 1). Algo semejante sucedió cuando se preguntó al personal sanitario si consideraban que los pacientes entendían lo que se les explicaba, donde las respuestas positivas aumentaron y disminuyeron las negativas $(p=0,026)$ (gráfico 2$)$.

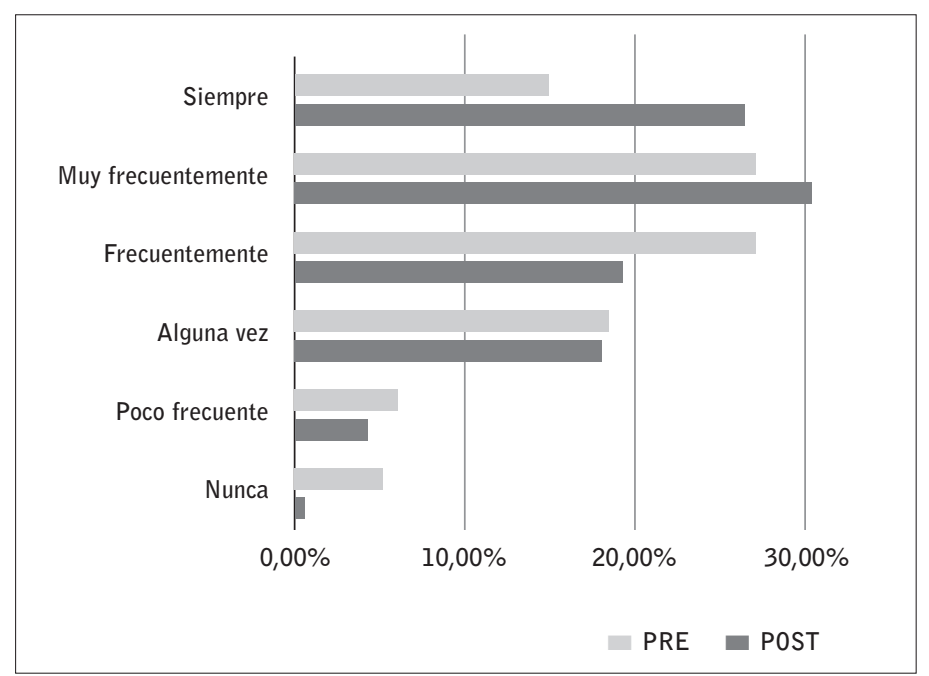

Gráfico 1. Porcentaje de personal que habla con los pacientes sobre acceeso vascular, dieta, cuidados... de forma regular. Comparación pre y post intervención.

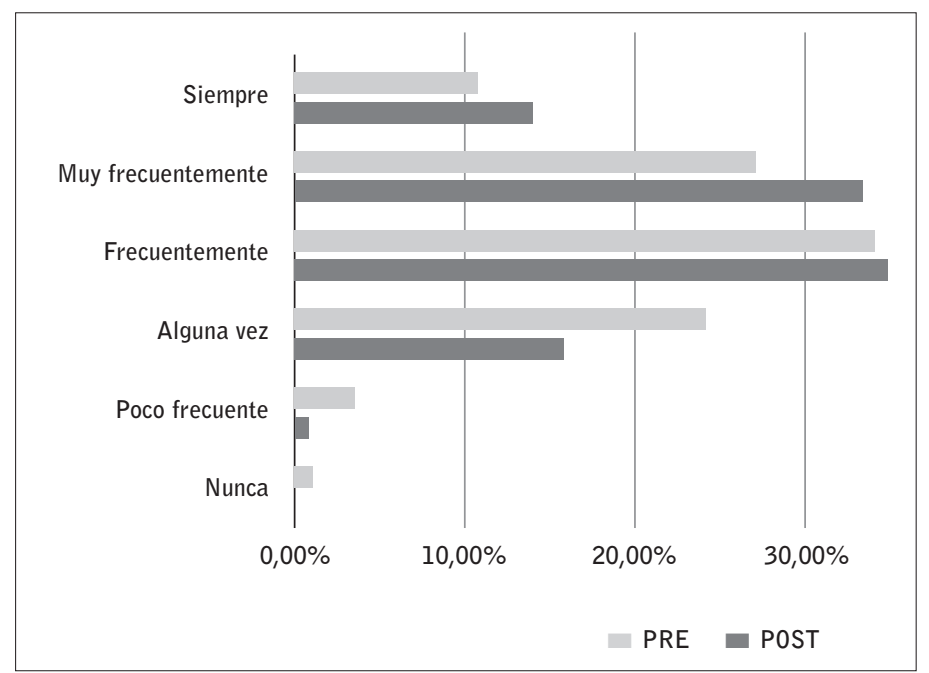

Gráfico 2. Porcentaje de personal que considera que los pacientes suelen entender lo que les explican. Comparación pre y post intevención. 


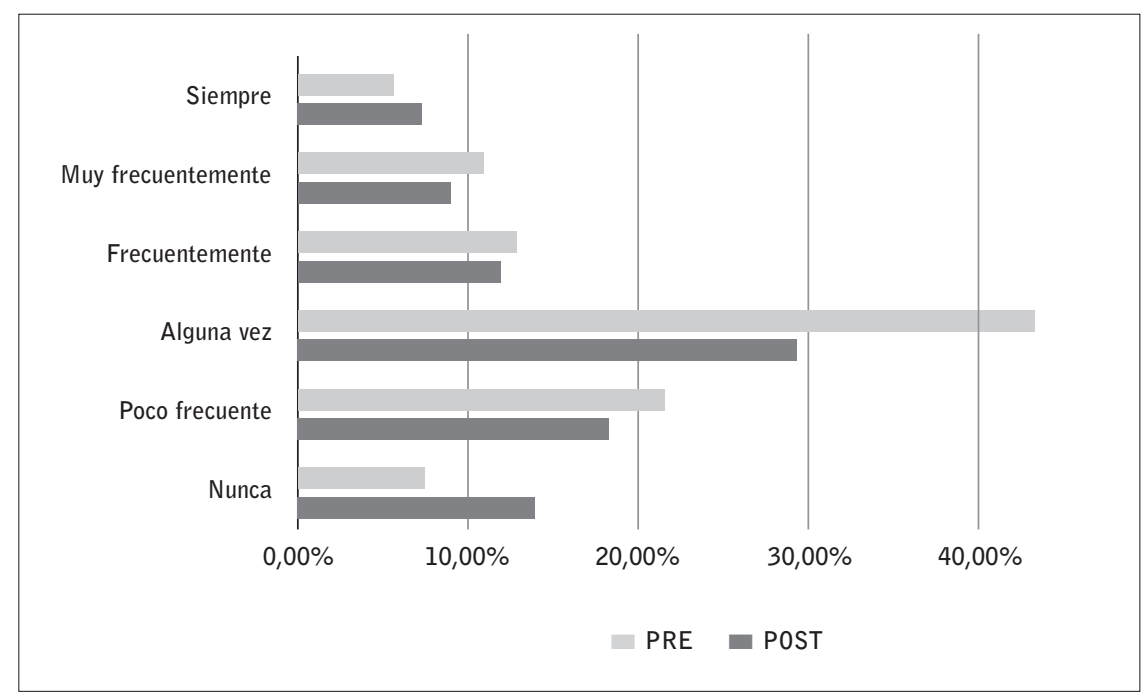

Gráfico 3. Porcentaje de personal que considera que podría ser beneficioso para e paciente que entraran familiares o amigos a las sesiones de hemodiálisis. Comparación pre y post intervención.

Otro de los resultados estadísticamente más significativos fue el que responde a la pregunta "¿Te sientes cercana a los pacientes durante todas las sesiones de diálisis?" donde, a pesar de la situación de pandemia que se estaba viviendo en esos momentos, se registró un aumento importante en los valores positivos "siempre" $y$ "frecuentemente" ( $p=0,002)$. Por otra parte, los profesionales también consideran que los pacientes se sienten cómodos durante las sesiones de hemodiálisis $(p=0,018)$.

Uno de los resultados más controvertidos es el obtenido en la pregunta "¿Consideras que podría ser beneficioso para el paciente que entraran familiares 0 amigos a las sesiones de diálisis?" donde, a pesar de no ser una diferencia estadísticamente significativa, las respuestas negativas (nunca y poco frecuentemente) aumentaron tras la implantación de las medidas de humanización y disminuyeron de forma notable las respuestas positivas (siempre, muy frecuentemente, frecuentemente y alguna vez) (gráfico 3).

Los profesionales sanitarios de los centros de la FRIAT consideran, teniendo en cuenta la percepción de la cultura de humanización, que es necesaria la formación en el ámbito de la humanización y creen que sería conveniente mejorar la humanización en las unidades de hemodiálisis $(p=0,000)$ (gráfico 4$)$.

Especial atención merecen los resultados obtenidos en las tres últimas preguntas del cuestionario a pacientes, las cuales fueron formuladas post intervención al estar enfocadas a una de las medidas de humanización que se implantó: acompañar en diálisis. La primera de las preguntas está relacionada con las visitas de familiares o personas de confianza del paciente a la unidad de hemodiálisis donde recibe tratamiento, una de las medidas que entró en vigor durante el proyecto y que resultó más novedosa y controvertida: "En mi unidad de diálisis se permite que pueda entrar un familiar o persona de confianza si se lo pido con tiempo y se puede hacer de forma ordenada" donde los porcentajes obtenidos para "siempre" y "nun$\mathrm{ca}^{\prime \prime}$ fueron prácticamente iguales $(39,2 \%$ versus $39,6 \%)$.

Estos datos concuerdan con los obtenidos en la pregunta "Considero importante que pueda entrar algún familiar o persona de confianza a acompañarme un rato durante la diálisis" donde el 34,4\% consideran que siempre es importante, un $28 \%$ creen que sería importante alguna vez y el $37,6 \%$ lo consideran como algo nunca importante.

La pregunta: "Sería interesante poder hacer videollamadas con discreción durante la sesión de diálisis a familiares o personas cercanas" también fue incluida en el cuestionario a pacientes post intervención a raíz de la

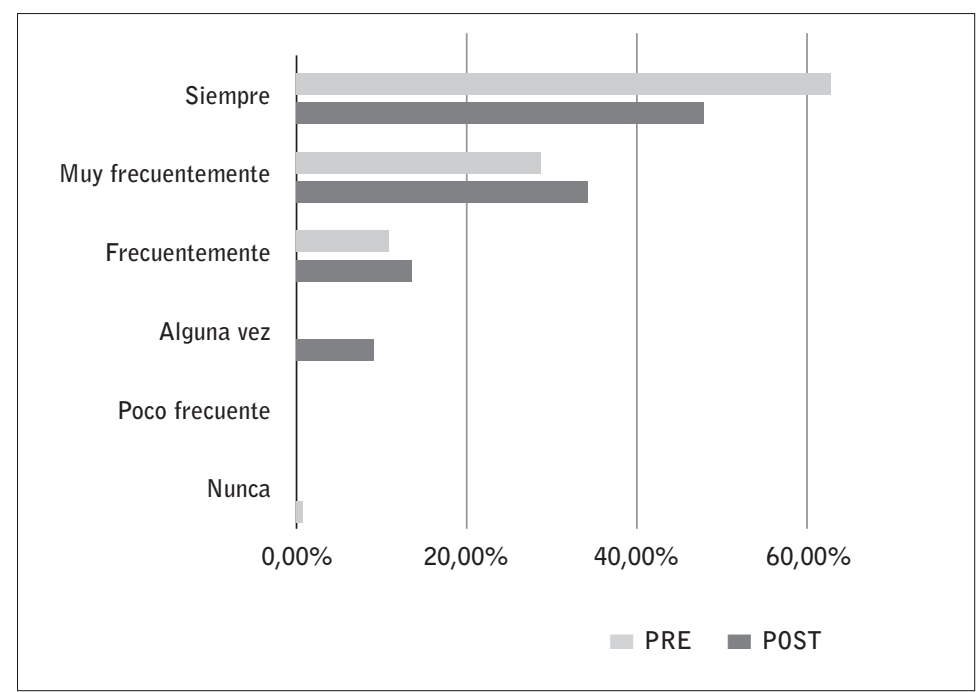

Gráfico 4. Porcentaje de personal que considera necesaria la formación en humanización en la hemodiálisis. Comparación pre y post intervención. 
pandemia COVID-19 y los resultados obtenidos fueron favorables, con un $41,1 \%$ que opinan que siempre sería interesante y un 30,5\% que opinan que lo sería alguna vez.

El resto de resultados registrados en los cuestionarios, tanto de personal como de pacientes, no obtuvieron respuestas con un peso estadístico en el estudio, al realizar la comparación pre y post intervención.

\section{Discusión}

Tras la implantación de las medidas de humanización en los centros, los pacientes aumentaron su grado de satisfacción en diferentes ámbitos: la información recibida de los procesos realizados durante la sesión de hemodiálisis, los aspectos o temas que les generan inquietudes y que pueden suponer una problemática importante en el desarrollo o la evolución de su enfermedad (control de la dieta, cuidados que precisan una fístula arteriovenosa, un catéter venoso central...). Estos resultados se ajustan a los obtenidos en los cuestionarios de los profesionales sanitarios, donde se refleja el aumento en el número de personas que llevan a cabo esas intervenciones educativas y que sienten que están teniendo un impacto positivo en sus pacientes.

Por otro lado, la revisión bibliográfica de Romero-Ruiz et al. ${ }^{7}$ muestra un elevado grado de insatisfacción de los pacientes acerca de la información relacionada con la medicación, la dieta, etc. Lo cual refuerza aún más la importancia de llevar a cabo acciones formativas como las implantadas en las unidades a lo largo del desarrollo del presente proyecto.

La información es una pieza clave en la tranquilidad y satisfacción de los pacientes, una persona que está informada es una persona cuyo grado de ansiedad frente a lo desconocido disminuye, generando un mayor grado de satisfacción con su alrededor, y en este caso, con los profesionales sanitarios que los rodean. La comunicación del equipo de salud con los pacientes es una parte fundamental de los cuidados humanizados. Un profesional que responde a las preguntas de sus pacientes, que les informa previamente a realizar cualquier intervención o procedimiento y que les resuelve sus dudas, equivale a un paciente satisfecho, se reducen los conflictos y malentendidos y aumenta la confianza de estos en los profesionales que les atienden ${ }^{8-13}$.

Además de las intervenciones educativas realizadas con los pacientes, otra medida de humanización implantada fue el acompañamiento en diálisis. Este novedoso y pionero proyecto en las unidades de hemodiálisis pretende ofrecer un acompañamiento por parte de una persona cercana al paciente, ya sea un familiar, amigo o persona de confianza, que permita disminuir los niveles de ansiedad y de temor, y haga de la sesión de hemodiálisis un tiempo lo más agradable y confortable posible y no un "castigo" para la persona que lo recibe. Los resultados obtenidos en el cuestionario post intervención acerca de estas visitas de los familiares a las unidades muestra que el $39,2 \%$ de las unidades de HD permitía el acceso a familiares o conocidos de los pacientes durante la sesión y el $39,6 \%$ no lo hacía. Estos resultados se equiparan en gran medida con los de otros estudios y proyectos a nivel nacional, como el conocido Proyecto Humanizando las Unidades de Cuidados Intensivos (HUCI), donde se mostró que, a pesar de los esfuerzos y recomendaciones existentes en la literatura ${ }^{14}$ para crear unidades de cuidados intensivos de puertas abiertas, todavía son muchas las unidades que se resisten a este cambio, lo cual alerta a la comunidad sobre la situación de la humanización en la atención sanitaria en este aspecto y la importancia de concienciar a la población y sobre todo a las autoridades de que las visitas a los pacientes son una necesidad. EI planteamiento de esta medida consiste en que el acompañante, solicitado por el paciente, para entrar a la sala de diálisis, lo haga de forma organizada, controlada, con un tiempo limitado, de forma puntual, sin molestar a otros pacientes y firmando un consentimiento informado donde se detalla el cumplimiento de unas normas y el abandono de la sala cuando así se le solicite.

Hay que tener en cuenta que este proyecto se ha visto afectado, al igual que tantos otros, por la pandemia de la COVID-19, situación en la que los centros se vieron obligados a prohibir el acceso a personas ajenas al mismo por motivos de seguridad. Este hecho motivó que la pregunta sobre la posibilidad de efectuar videollamadas durante la sesión obtuviera mejores resultados, donde el 71,6\% de los pacientes estaría dispuesto a hacerlas para poder comunicarse con sus seres queridos durante el desarrollo de la misma.

Destacar que tal y como se mostró en los resultados, el $62,4 \%$ de la muestra de pacientes considera importante en mayor o menor medida la posibilidad de realizar esas visitas durante la sesión de HD, por lo que podría tratarse de una medida con un importante futuro por delante.

Las visitas no sólo son un apoyo para el paciente, también lo son para sus familiares y seres queridos, a los que se les brinda la posibilidad de conocer de primera 
mano el funcionamiento de la unidad, el personal que en ella trabaja, preguntar y dar solución a dudas sobre el tratamiento, lo cual resulta de primera necesidad para estas personas, según se recoge en la bibliografía $^{15,16}$. La familia desempeña un papel fundamental en el cuidado de la persona enferma, por lo que es de vital importancia que se les incluya en el tratamiento y que puedan acompañar a su ser querido durante la sesión de HD14,15. Se ha demostrado que el hecho de que los familiares o personas cercanas a los pacientes puedan acompañar a su ser querido durante su hospitalización, disminuye los niveles de ansiedad, ayuda a incrementar el bienestar del paciente y minimiza la experiencia traumática de la enfermedad, tanto del paciente como de la persona que lo acompaña ${ }^{14}$.

Como se expuso en el apartado de resultados, las respuestas obtenidas por parte de los profesionales sanitarios respecto a la importancia del acompañamiento en diálisis fueron más negativas de lo esperado, lo cual muestra una evidente resistencia al cambio, frecuentemente asociada a lo desconocido, a la implantación de medidas que nunca antes se habían Ilevado a cabo y que, en cierta medida, modifican el trabajo diario. En ocasiones, creencias por parte de los profesionales como que las visitas interfieren o retrasan la aplicación de cuidados y el correcto desarrollo del tratamiento, limitan en gran medida la aceptación de medidas como la implantada en este proyecto de humanización. Parece existir un sentimiento de propiedad y control del entorno de la sala por parte del personal respecto a terceras personas, las cuales no tienen por qué afectar a la asistencia sanitaria siempre y cuando cumplan con las medidas higiénicas de seguridad y atiendan a las consignas establecidas.

No se puede olvidar que la enfermería es una profesión implícitamente humana, basada en las relaciones con el paciente y su cuidado. Watson (1979), autora de la Teoría del Cuidado Humanizado decía: "el cuidado es la esencia de la práctica de enfermería, la empatía, la comprensión e intencionalidad son factores fundamentales para que los profesionales de enfermería y sus pacientes formen un vínculo humano que trascienda la medicalización y el control terapéutico'117. Durante el proceso de enfermedad y el tratamiento, en este caso, de una enfermedad crónica, la enfermera genera un vínculo con sus pacientes que le permite conectar emocionalmente y ofrecer unos cuidados holísticos a la persona enferma. Esta es la razón por la cual, a pesar de la actual pandemia COVID-19, las enfermeras y demás profesionales sanitarios no han perdido su esencia y, a pesar de la distancia social, de los equipos de protección individual y de las medidas de aislamiento, se mantienen cercanas a sus pacientes. Simples gestos como dar los buenos días, llamar a la persona por su nombre, o darles la mano cuando lo necesitan, suponen para los pacientes el apoyo y la fuerza para seguir adelante. Tanto es así que, a pesar de la situación, los resultados obtenidos en los cuestionarios registraron un aumento estadísticamente significativo en los valores positivos "siempre" $y$ "frecuentemente" cuando se preguntaba a los profesionales si se sentían cercanos a sus pacientes durante las sesiones de HD. Esto concuerda con las respuestas obtenidas en los cuestionarios de los pacientes, analizadas en apartados anteriores, y en diversos estudios donde se muestra que un trato cercano, cálido y que genera un ambiente de confianza y seguridad, influirá de manera positiva en las relaciones entre el profesional y el paciente y aumentará la satisfacción de los segundos 7,13,18.

Otra de las medidas de humanización que se implantó en el desarrollo de este proyecto, fue la humanización de la infraestructura. Según Sousa Kayo et al. las estructuras físicas inadecuadas son un obstáculo para el cuidado humanizado, ya que son el espacio donde el paciente debe permanecer mientras recibe su tratamiento ${ }^{11}$. Espacios amplios, con decoración agradable (dibujos coloridos, renovación de mobiliario y pintura), con ventanas que permitan la entrada de luz natural, renovación de sillones autorregulables, instalación de redes wifi, temperatura confortable, lugares donde guardar de forma segura sus pertenencias mientras se recibe el tratamiento, etc. ${ }^{11,18}$ conseguirán que las salas de HD sean lugares cálidos y acogedores donde el paciente se sienta cómodo durante el desarrollo de su tratamiento.

Tan importante es una infraestructura humanizada, como la formación en este ámbito de los profesionales que en él trabajan. Los resultados de la presente investigación ponen de manifiesto que los profesionales sanitarios de los centros de la FRIAT consideran necesaria la formación en el ámbito de la humanización y creen que sería conveniente mejorar la humanización en las unidades de hemodiálisis, datos que coinciden de nuevo con las conclusiones del estudio de Sousa Kayo ${ }^{11}$.

Como principal limitación del estudio destacar que la pandemia de la COVID-19 supuso una situación en la que, por motivos de seguridad, se paralizaron muchas de las medidas de humanización que se estaban 
implantando en ese momento en los centros, lo cual interfirió en los resultados de los cuestionarios post intervención.

Los resultados obtenidos en la presente investigación muestran una buena acogida del proyecto de humanización tanto por parte de los pacientes como de los profesionales que trabajan en sus centros. Se ha visto un incremento estadísticamente significativo en la satisfacción de los pacientes respecto a la información obtenida por los profesionales, la cercanía de éstos y, aunque la comparación pre y post intervención no resultó significativa en algunos ítems, se registraron valores muy positivos respecto al trato que reciben los pacientes y la comodidad de éstos en los centros. No obstante, se observó que los profesionales sanitarios de los centros de la FRIAT consideran necesaria la formación en el ámbito de la humanización y creen que sería conveniente mejorar ésta en las unidades de HD.

Todas las unidades de HD pueden mejorar la atención humanizada a sus pacientes renales mediante una formación al personal, motivación del mismo e inversiones por parte de las instituciones en implantar todas las medidas de humanización que estén a su alcance. Dice Eric Casell "Los que sufren no son los cuerpos sino las personas 1129 por tanto, demos a las personas el trato humano que se merecen, seamos profesionales excelentes tanto a nivel científico-técnico como humano.

\section{Agradecimientos}

Expresar nuestro más sincero agradecimiento por su colaboración a todos los pacientes renales y profesionales sanitarios de la Nefrología que han participado en este estudio, así como a todas aquellas personas que han hecho posible que este proyecto salga adelante.

Recepción: 03-06-21

Aceptación: 25-07-21

Publicación: 30-09-21

\section{Bibliografía}

1. Mijangos-Fuentes KI. El paradigma holístico de la enfermería. Salud y Administración [Internet] 2014 [consultado 08 Sep 2021];1(2):17-22. Diponible en: https://revista.unsis.edu.mx/index.php/saludyadmon/ article/view/86/83.

2. Monje $P$, Miranda P, Oyarzun J, Seguel F, Flores E. Percepción del cuidado humanizado de enfermería desde la perspectiva de usuarios hospitalizados. Ciencia y Enfermería [Internet]. 2017 [consultado 20 0ct 2019]; 24(5):43-52. Disponible en: https://scielo.conicyt.cl/ pdf/cienf/v24/0717-9553-cienf-24-5.pdf.

3. Prías Vanegas HE. Cuidado humanizado, un desafío para el profesional de enfermería. REVISALUD Unisucre [Internet]. 2017 [consultado 02 Sep 2020]; 3(1):26-30. Disponible en: https://www.recia.edu.co/ index.php/revisalud/article/view/575/620.

4. Federación Española de Empresas de Tecnología Sanitaria. Impacto de las tecnologías sanitarias aplicadas a los tratamientos de diálisis en el SNS. Madrid: Fenin. 2018.

5. Consejería de Sanidad de la Comunidad de Madrid. Plan de Humanización de la Asistencia Sanitaria 20162019. [Internet]. Madrid: Dirección General de Coordinación de la Atención al Ciudadano y Humanización de la Asistencia Sanitaria; 2016 [consultado 28 0ct 2019]. Disponible en: http://www.madrid.org/bvirtual/ BVCM017902.pdf.

6. Pedreira-Robles G, Vasco-Gómez A, Martínez-Delgado $Y$, Herrera-Morales C, Baz-Rodríguez MT, Junyent-Iglesias $\mathrm{E}$. Déficit de actividades recreativas en hemodiálisis. Satisfacción y coste económico ante un proyecto lúdico terapéutico. Enferm Nefrol. 2020 Jan; 23(1):83-92.

7. Romero-Ruiz AB, Sevillano-Jiménez A, Cardador-Trócoli $C$. Factores asociados a la satisfacción del paciente en diálisis. Enferm Nefrol. 2019 Abr-Jun; 22(2):11223.

8. Shah S, Patel A, Rumoro DP, Hohmann S, Fullam F. Managing patient expectations at emergency department triage. PXJ: Patient Experience Journal. 2015 [consultado 06 Jun 2021]; 2(2):31-44. Disponible en: https://pxjournal.org/journal/vol2/iss2/6/. 
9. Abolfotouh MA, Al-Assiri MH, Alshahrani RT, Almutairi ZM, Hijazi RA, Alaskar AS. Predictors of patient satisfaction in an emergency care centre in central Saudi Arabia: a prospective study. Emerg Med J. 2017 [consultado 08 Jun 2021]; 34:27-33. Disponible en: https:// emj.bmj.com/content/emermed/34/1/27.full.pdf.

10. Messina G, Vencia F, Mecheroni S, Dionisi S, Baragatti L, Nante N. Factors affecting patient satisfaction with emergency department care: an Italian rural hospital. Glob J Health Sci. 2015 [consultado 08 Jun 2021]; 7(4):30-9. Disponible en: http://www.ccsenet.org/journal/index.php/gjhs/article/view/42056.

11. Sousa KHJF, Damasceno CKCS, Almeida CAPL, Magalhães JM, Ferreira MA. Humanization in urgent and emergency services: contributions to nursing care. Rev Gaucha Enferm. 2019 [consultado 06 Jun 2021]; 40:e20180263. Disponible en: https:// www.scielo.br/j/rgenf/a/PX7vJwFyrRTsVm3jgMk8rRN/? format=pdf\&lang=en.

12. Romero-García $M$, Delgado-Hito $P$, de la Cueva-Ariza L, Martínez-Momblán MA, Llunt-Canut MT, Trujols-AIbet $\mathrm{J}$, et al. Level of satisfaction of critical care patients regarding the nursing care received: Correlation with sociodemographic and clinical variables. Australian Critical Care [Internet] 2019. [consultado 17 Jun 2021] 32 (1) 483-96. Disponible en: https://reader. elsevier.com/reader/sd/pii/S103673141830184X?token=19096A0A73EC216D843CDE7A7D8223A 52371B46658270CF56068E406D20A1D55E8B5B2550B3E4D15A51C7D55146CE3CA\&originRegion=eu-west-1\&originCreation=20210617092305.

13. Sanz-Turrado M, Garrido-Pérez L, Caro-Domínguez C. Factores que influyen en la satisfacción del paciente de diálisis con enfermería. Enferm Nefrol. 2017: enero-marzo; 20(1):66-75.
14. Escudero D, Martín L, Viña L, Forcelledo L, García-Arias B, López-Amor L. Abrir las puertas de la UCI. Una necesidad inexcusable. Medicina Intensiva [Internet] 2015 Nov [consultado 27 May 2021]; 39(8):522-3. Disponible en: https://www.medintensiva.org/es-abrir-puertas-uci-una-necesidad-articulo-S0210569115001485.

15. De la Cueva-Ariza $L$, Delgado-Hito $P$, Martínez-Estalella G, Via-Clavero G, Llunch-Canut T, Romero-García M. Implementación de la evidencia para la mejora del cuidado de enfermería a la familia del paciente crítico: una Investigación Acción Participativa. BMC Health Serv Res [Internet] 2018 [consultado 26 May 2021]; 18:357. Disponible en: https://bmchealthservres.biomedcentral.com/articles/10.1186/s12913-018-31778 8\#citeas.

16. Santamaría NP, Rodríguez KA, Carrillo GM. Percepción del comportamiento de cuidado de enfermería en adultos con terapia renal de diálisis peritoneal y hemodiálisis. Enferm Nefrol. 2019 Jul-Sep;22(3):284-92.

17. Urra E, Jana A, García M, Algunos aspectos esenciales del pensamiento de Jean Watson y su teoría de cuidados transpersonales. Ciencia y enfermería [Internet] 2011 [consultado 06 Jun 2021]; XVII(3):11-22. Disponible en: https://scielo.conicyt.cl/pdf/cienf/v17n3/art02.pdf.

18. Grupo de trabajo de certificación de Proyecto HU-CI. Manual de buenas prácticas de humanización en Unidades de Cuidados Intensivos. Madrid: Proyecto HUCI; 2019 [consultado 09 Jun 2021]. Disponible en: https://proyectohuci.com/wp-content/uploads/2019/05/ Manual_BP_HUCI_rev2019_web.pdf.

19. Cassell EJ.The nature of suffering and the goals of medicine N Engl J Med. 1982 Mar 18;306(11):639-45 doi: 10.1056/NEJM198203183061104. 


\section{Anexo 1. LÍNEAS DE ACTUACIÓN DEL PROYECTO HUMANIZANDO LA HEMODIÁLISIS}

- Destacar el rol de la Enfermería Nefrológica en la Humanización

- Mejorar la comunicación con el paciente durante las sesiones de HD

- Potenciar la importancia de la acogida a la unidad en las primeras diálisis

- Organización talleres formativos durante las sesiones

1. Cuidados del acceso vascular

2. Dietas

3. Características del tratamiento ...

- Comunicación con las familias y acceso a las salas de hemodiálisis

- Fomentar el entretenimiento durante la sesión
1. Lectura
2. Juegos de mesa
3. Ejercicio
4. Facilitar alguna actividad laboral
5. Propiciar el descanso y sueño si es necesario

- Otras acciones encaminadas a personalizar operativas o estratégicas sobre humanización en hemodiálisis

- Otras acciones encaminadas a personalizar operativas o estratégicas sobre humanización en hemodiálisis

- Adecuar los espacios, infraestructuras y equipamientos para un mayor confort

- Significar eventos personales como los cumpleaños

- Envío de cartas de duelo, firmadas por los trabajadores, a los familiares de pacientes que hayan causado exitus

- Participar en celebraciones festivas

- Disponer de sistemas de opinión de los usuarios

- Integrar la asistencia de personal médico y de enfermería

- Rentabilizar el tiempo de diálisis para consultas o controles

- Elaborar y difundir un Decálogo para la humanización de las unidades de hemodiálisis

- Difundir el proyecto de humanización y sus medidas a los trabajadores de la FRIAT para reforzar su implantación desde el departamento de Comunicación

- Dar a conocer al resto de la Nefrología española el Proyecto mediante participaciones en Congresos o Seminarios, así como intentar su publicación en Enfermería Nefrológica, para poder compartir las experiencias y servir de ayuda en la adopción de sus medidas en cualquier unidad de diálisis 


\section{Anexo 2. HUMANIZANDO LA HEMOdIÁLISIS. DECÁLOGO PARA LOS PROFESIONALES DESDE LA PERSPECTIVA DEL PACIENTE.}

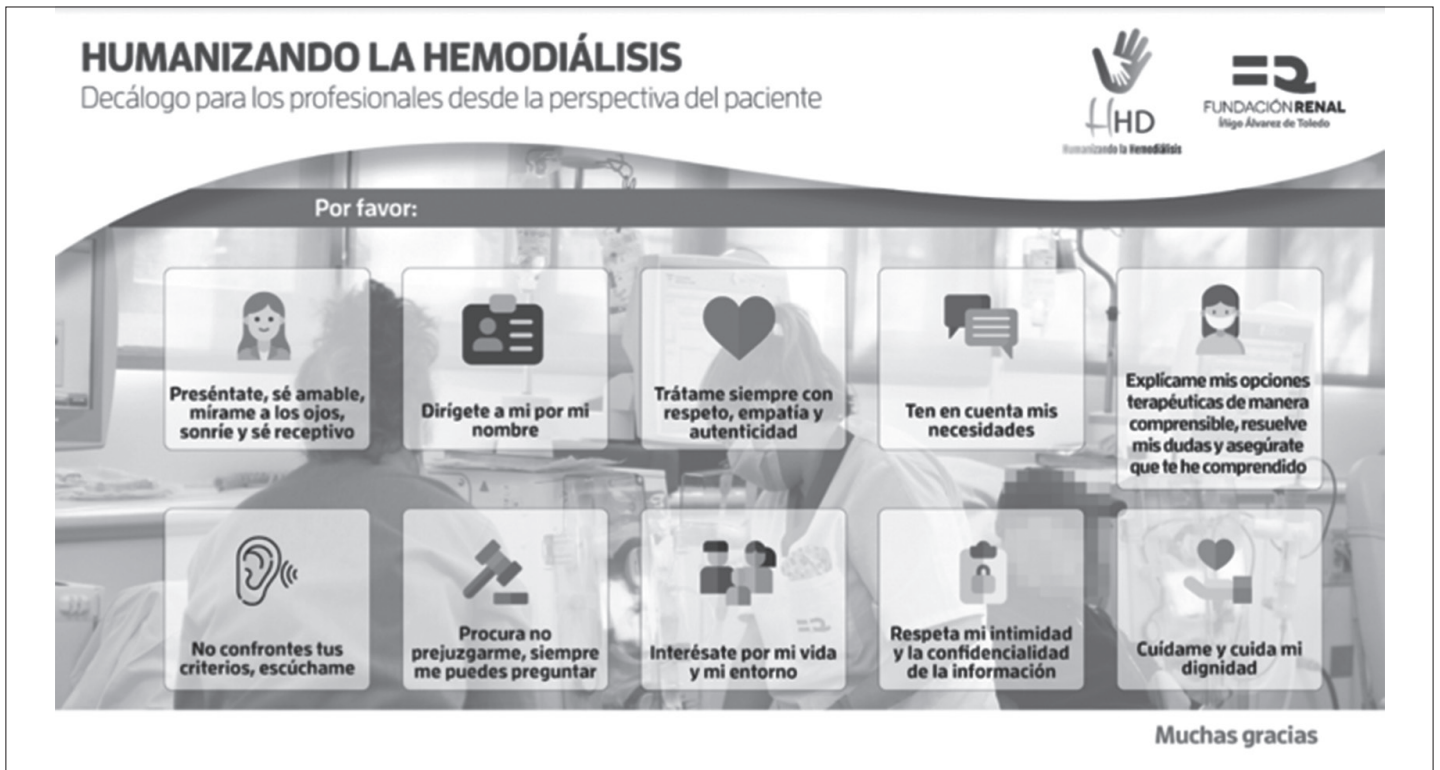




\section{Anexo 3. CUESTIONARIO VALORACIÓN DE LA ATENCIÓN HUMANIZADA-PACIENTES.}

\section{VALORACIÓN DEL PACIENTE DE LA ATENCIÓN HUMANIZADA}

$\square$ Al marcar esta casilla acepto participar de forma voluntaria en el Proyecto Humanizando la Hemodiálisis impulsado por la Fundación Renal Íñigo Álvarez de Toledo. Doy así mismo mi consentimiento para el uso, tratamiento y posterior explotación y difusión de la información obtenida en el estudio, siempre que se realice de forma anónima y conforme a la vigente Ley de Protección de Datos, con pleno derecho a revocar mi consentimiento en cualquier momento si así lo considerara necesario.

Por favor califique las siguientes cuestiones sobre el cuidado y trato del personal de la Unidad durante sus diálisis.

Sólo hay una respuesta para cada pregunta.

- El personal sabe qué es lo que me hace falta durante la diálisis

- El personal conoce mis gustos y preferencias

- El personal se esfuerza en que esté cómodo, atendido e incluso distraído durante la diálisis

- El personal me saluda cuando llego y cuando me marcho

- El personal me explica o informa de los procesos que se me realizan en la diálisis

- El personal conoce a mis familiares que se han pasado a verme a la diálisis

- El personal me dedica el tiempo que necesito

- Me siento escuchado por el personal cuando les cuento cosas o necesidades

- El personal intenta satisfacer mis necesidades durante la diálisis

- Obtengo la información que necesito cuando le pregunto al personal

- El personal sabe cuánto tiempo llevo en diálisis y la evolución de mi enfermedad

- El personal respeta mi intimidad y mis decisiones

- Las instalaciones de la Unidad de diálisis son agradables y confortables

- El personal me pregunta se interesan por cómo me encuentro

- El personal se interesa por lo que me ha pasado o qué tal he estado los días antes de la diálisis

- El personal me pregunta sobre mi dieta, si hago deporte, si tomo la medicación, etc.

- El personal me pregunta si tengo alguna sugerencia para mejorar mi bienestar

- El personal me llama por mi nombre

- Me siento tratado con respeto e incluso con cariño y cercanía

- El personal usa el teléfono para asuntos personales en la sala de diálisis

- El personal me cuenta las cosas

para que las entienda y de forma positiva

- El personal suele quedarse en los controles de Enfermería durante la diálisis

- El personal ayuda para coordinar mis necesidades de vacaciones, papeleos o consulta con el psicólogo

- Considero importante que pueda entrar algún familiar o persona de confianza a acompañarme un rato durante la diálisis 
- En mi unidad de diálisis permiten que pueda entrar un familiar o persona de confianza si se lo pido con tiempo y se puede hacer de forma ordenada

- Sería interesante poder hacer videollamadas con discreción durante la sesión de diálisis a familiares o personas cercanas

\section{GENERAL}

Género: $\quad \square$ Hombre $\quad \square$ Mujer

Edad en años:

Estado civil: $\square$ Soltero $\square$ Casado/en pareja $\square$ Separado/divorciado $\square$ Viudo

¿Cuánto tiempo lleva en diálisis aproximadamente?

Tiempo en años:

En general, ¿cómo calificaría su salud?

$\square$ Excelente $\square$ Buena $\quad \square$ Normal $\square$ Mala $\square$ Muy mala $\square$ No está seguro

¿Qué aspectos mejoraría de la atención recibida por el personal? 


\section{Anexo 4. CUESTIONARIO VALORACIÓN DE LA ATENCIÓN HUMANIZADA - PROFESIONALES.}

\section{CUESTIONARIO DE EVALUACIÓN HUMANIZANDO LA HEMODIÁLISIS}

\section{CUESTIONARIO:}

Responde a cada pregunta del 1 al 6 en función del siguiente baremo:

1- Nunca

2- Poco frecuente

3- Alguna vez

4- Frecuentemente

5- Muy frecuentemente

6- Siempre

1. ¿Consideras necesario incluir la cultura de humanización en la práctica habitual de los cuidados del paciente renal?
$\square 1 \quad \square 2$
$\square 3 \square$
$\square 5 \square 6$

2. ¿Tienes la posibilidad de saludar a todos los pacientes en diálisis de tu turno?

$\square 1 \quad \square 2 \quad \square 3 \quad \square 4 \quad \square 5 \quad \square 6$

3. ¿Consideras en general que los pacientes están cómodos durante las sesiones de HD?
$\square 1$
$\square 2$
$\square 3$
$\square 4$
$\square 5 \square 6$

4. ¿Sueles conocer algo de lo que comen y beben los pacientes cuando no están en diálisis?
$\square 1$
$\square 2$
$\square 3$
$\square 4 \quad \square 5$
$\square 6$

5. ¿Consideras que podría ser beneficioso para el paciente que entraran familiares o amigos a las sesiones de diálisis?

$\square 1 \quad \square 2 \quad \square 3 \quad \square 4 \quad \square 5 \quad \square 6$

6. ¿Hablas con los pacientes sobre AV,dieta, cuidados, etc., de forma regular?

$\square 1 \quad \square 2 \quad \square 3 \quad \square 4 \quad \square 5 \quad \square 6$

7. ¿Consideras que los pacientes suelen entender lo que les explicas?
$\square 1 \quad \square 2$
$\square 3 \square 4$
$\square 5 \quad \square 6$

8. ¿Conoces la situación personal de la mayoría de los pacientes?

$\square 1 \quad \square 2 \quad \square 3 \quad \square 4 \quad \square 5 \quad \square 6$

9. ¿Te sientes cercana a los pacientes durante todas las sesiones de diálisis?
$\square 1$
$\square 2$
$\square 3$
$\square 4 \square 5$
$\square 6$

10. ¿Conoces el nombre de todos los pacientes de tu turno habitual?

$\square 1 \quad \square 2 \quad \square 3 \quad \square 4 \quad \square 5 \quad \square 6$




\section{1. ¿Consideras necesaria la formación en humanización en hemodiálisis?}

$\square 1 \quad \square 2 \quad \square 3 \quad \square 4 \quad \square 5 \quad \square 6$

12. ¿Crees que es conveniente mejorar la humanización en la hemodiálisis?
$\square 1$
$\square 2$
$\square 3$
$\square 4$
$\square 5$
$\square 6$

\section{PREGUNTAS GENERALES}

Sexo: $\square$ Hombre $\quad \square$ Mujer

Edad:

Categoría profesional : $\square$ Enfermería $\square$ Auxiliar $\square$ 0tro

Este artículo se distribuye bajo una Licencia Creative Commons Atribución-NoComercial 4.0 Internacional. https://creativecommons.org/licenses/by-nc/4.0/

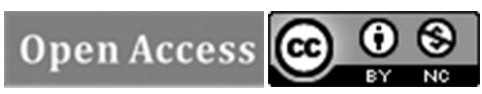

